\title{
Analyse comparative de la productivité des cheptels de petits ruminants en élevage extensif tropical : une nouvelle approche par les modèles matriciels en temps discret
}

\author{
M. Lesnoff ${ }^{1 *}$ R. Lancelot ${ }^{1,2}$ E. Tillard ${ }^{1,3}$ B. Faye ${ }^{1}$
}

\begin{abstract}
Mots-clés
Ovin - Dynamique des populations Productivité - Modèle mathématique Sénégal.
\end{abstract}

\begin{abstract}
Résumé
Une nouvelle méthode d'analyse comparative de la productivité des cheptels domestiques tropicaux est présentée ici. Cette méthode a utilisé les modèles démographiques matriciels et la méthode des modèles de production à l'équilibre (steady-state models). Les méthodes démographiques classiques utilisent des modèles à pas de temps annuel, peu adaptés pour les espèces à cycle de reproduction relativement court et dont les mises bas surviennent tout au long de l'année. Dans ce nouveau modèle, l'année a été décomposée en quinzaines. Trois apports opérationnels principaux ont été présentés. Premièrement, le pas de temps court a pu diminuer le biais dans l'estimation des paramètres démographiques (fécondité, mortalité, exploitation ou importation $\mathrm{d}^{\prime}$ animaux). Deuxièmement, le modèle périodique a pu représenter conjointement les variations intra-annuelles et interannuelles des paramètres démographiques et d'autres paramètres comme le poids ou le prix de vente des animaux. Enfin, la méthode d'inférence proposée (utilisant le bootstrap non paramétrique) a permis de calculer des intervalles de confiance et de réaliser des tests pour comparer la productivité de cheptels différents. La méthode a été testée avec des données de terrain récoltées sur des cheptels d'ovins au Sénégal. Elle peut également être appliquée à d'autres espèces domestiques ou sauvages dans divers contextes zootechniques ou écologiques.
\end{abstract}

1. Cirad-emvt, TA30/A, Campus international de Baillarguet, 34398 Montpellier Cedex 5, France

2. Adresse actuelle : Isra/Lnerv, BP 2057, Dakar-Hann, Sénégal

3. Adresse actuelle : Cirad, Station de Ligne-Paradis, 7 chemin de 1'Irat, LigneParadis, 97410 Saint-Pierre, Ile de la Réunion, France

* Auteur pour la correspondance

Tél. : +33 (0)4 67593770 ; fax : +33 (0)4 67593825

E-mail: matthieu.lesnoff@cirad.fr

\section{INTRODUCTION}

Les épidémiologistes et les zootechniciens tropicalistes ont besoin de méthodes permettant de quantifier la productivité d'un cheptel (définie ici par un ensemble d'animaux regroupés en un ou plusieurs troupeaux) pour réaliser le diagnostic d'un système de production, pour le comparer à d'autres systèmes ou pour évaluer l'efficacité d'interventions zootechniques ou sanitaires (modification d'un régime alimentaire, évaluation zoo-économique d'un vaccin ou d'un vermifuge, etc.). Divers indicateurs synthétiques de la productivité globale d'un cheptel ont été définis $(8,23,30,35$, $36,46,47)$. Les plus élaborés sont construits à partir de modèles démographiques qui représentent l'ensemble du cycle de vie des animaux. Ces modèles permettent de comprendre comment se construit la productivité globale d'un cheptel et d'identifier l'origine des différences observées entre cheptels (28). Une approche démographique classique correspond aux modèles de production à 
l'équilibre (steady-state models) $(4,38,46)$, mais d'autres sont possibles $(5,6,7,39)$.

Les modèles de production à l'équilibre font partie des modèles matriciels en temps discret introduits par Leslie (26) et développés par la suite $(12,45)$. Ils bénéficient à ce titre de nombreux résultats théoriques. Le principe consiste à diviser le cheptel étudié en catégories définies, par exemple, par le sexe et la classe d'âge des animaux. L'état du cheptel au temps $t$ est décrit par un vecteur $\mathrm{x}(\mathrm{t})$ dont les composantes sont les effectifs d'animaux par catégorie. Les animaux d'une même catégorie sont supposés réagir de manière identique à leur environnement. Une équation matricielle $\mathrm{x}(\mathrm{t}+1)=\mathrm{A} x(\mathrm{t})$ prédit par récurrence l'effectif dans chaque catégorie aux temps $t+1, t+2$, etc. La matrice de projection démographique A contient les paramètres démographiques (fécondité, mortalité naturelle, exploitation et importation dans les troupeaux) pour les différentes catégories d'animaux.

Dans ces modèles, la productivité numérique d'un cheptel est souvent définie comme l'effectif des animaux exploitables chaque année en situation d'équilibre démographique stationnaire (le cheptel est en situation d'équilibre stationnaire lorsque son état est identique en début et en fin d'année). Elle correspond au potentiel productif du cheptel pour un effectif total donné et certaines hypothèses concernant le niveau d'exploitation des différentes catégories d'animaux. Les productivités pondérales ou monétaires peuvent être obtenues en pondérant l'effectif des animaux exploités dans chaque catégorie par le poids ou le prix de vente moyen des animaux des catégories considérées. L'équilibre stationnaire est une hypothèse de travail pour le calcul de la productivité (d'autres hypothèses de croissance démographique peuvent néanmoins être posées sans difficulté ; cf. la partie discussion). Cette hypothèse ne correspond pas à un objectif de production mais permet de comparer plusieurs cheptels sur une base commune (46).

Dans les applications zootechniques usuelles, les modèles de production à l'équilibre sont construits en considérant un pas de temps annuel. L'état d'un cheptel en fin d'année est prédit à partir de son état initial à l'aide de paramètres démographiques annuels moyens. Chez les petits ruminants en Afrique subsaharienne, cependant, l'alternance de saisons sèche et pluvieuse provoque des stress périodiques et des variations des performances zootechniques. Les abattages massifs lors des fêtes religieuses, les ventes ou les confiages d'animaux entre éleveurs à certaines périodes de l'année provoquent de fortes variations dans les taux d'exploitation $(16,17,34)$. Le pas de temps annuel ne permet pas de représenter ces phénomènes.

L'objectif de cet article a été de présenter un modèle de production à l'équilibre construit sur la base d'un pas de temps plus court, prenant en compte les variations intra-annuelles des paramètres démographiques des cheptels. Ce modèle a décomposé l'année en phases, des quinzaines dans le cas présenté, et a prédit l'évolution du cheptel de phase en phase (27). Il est appelé « périodique » (12, 40) lorsque la matrice de projection annuelle A est constante d'année en année. Bien que la productivité numérique annuelle soit définie de la même manière que pour un modèle à pas de temps annuel, la méthode conventionnelle de calcul (46) ne peut s'appliquer au modèle par quinzaine en raison de la structure plus complexe de la matrice A. Une nouvelle méthode développée pour pallier cette difficulté est présentée ici $(28,29)$. Pour simplifier l'exposé, les principes ont été introduits en prenant l'exemple d'un modèle à pas de temps annuel. Les extensions pour le cas du modèle matriciel par quinzaine sont ensuite présentées.

A titre d'exemple, cette méthode a été appliquée pour évaluer l'efficacité zoo-économique d'un plan de prophylaxie de la pathologie respiratoire et du parasitisme gastro-intestinal chez les ovins Djallonké de la région de Kolda (Sénégal). Les différents avan- tages et les conditions d'utilisation du modèle proposé sont discutés à la fin de l'article.

NOUVELLE METHODE DEMOGRAPHIQUE POUR L'ANALYSE COMPARATIVE DE LA PRODUCTIVITE DE CHEPTELS

\section{Principe de la méthode pour un modèle à pas de temps annuel}

\section{Le cheptel et ses paramètres démographiques}

Pour présenter la méthode, nous avons pris l'exemple d'un cheptel qui contenait uniquement des femelles et dans lequel nous avons supposé qu'il n'y avait pas eu d'importation d'animaux. Dans les sections suivantes, nous avons précisé comment la méthode a pris en compte les mâles et les importations d'animaux.

Nous avons supposé que ce cheptel était décomposé en trois classes d'âge :

1. Ages exacts inférieurs ou égaux à un an (classe notée « 0-1 an »);

2. Ages exacts compris entre un an (exclu) et deux ans (classe notée « 1-2 ans »);

\section{Ages exacts supérieurs à deux ans (classe notée « $>2$ ans »).}

Dans le modèle, l'état du cheptel à un temps $t$ est décrit par les effectifs $x_{i}(t)$ des femelles présentes dans les classes d'âge $i$ ( $\mathrm{i}=1,2$ ou 3 ). Le cheptel est également décrit par un ensemble de paramètres démographiques pour chaque classe d'âge $\mathrm{i}$ :

- la fécondité $\mathrm{f}_{\mathrm{i}}$ représente l'effectif moyen de produits femelles (dans l'exemple présent, seule l'évolution du cheptel des femelles a été représentée, les naissances de mâles n'ont donc pas été comptabilisés) dans l'année issus d'une femelle appartenant à la classe i en début d'année ;

- la probabilité de survie dans le troupeau $\mathrm{s}_{\mathrm{i}}$ représente la probabilité de ne pas sortir du troupeau durant l'année pour une femelle dans la classe i en début d'année. Cette probabilité a été définie par $\mathrm{s}_{\mathrm{i}}=1-\mathrm{P}_{\mathrm{i}}(\mathrm{mor})-\mathrm{P}_{\mathrm{i}}(\exp )$, où $\mathrm{P}_{\mathrm{i}}(\mathrm{mor})$ et $\mathrm{P}_{\mathrm{i}}(\exp )$ ont représenté respectivement les probabilités de mort naturelle et d'exploitation (abattage, vente, confiage, etc.) dans l'année. Cette définition de la probabilité $s_{i}$ est discutée plus loin. Dans les cheptels domestiques, les naissances sont étalées dans l'année. Une « classe d'âge » supplémentaire (classe notée « 0 ») représente ainsi les femelles nées en cours d'année ; le paramètre $\mathrm{s}_{0}=1-\mathrm{P}_{0}($ mor $)-\mathrm{P}_{0}(\exp )$ représente la probabilité de ne pas sortir du troupeau avant la fin de l'année pour ces femelles.

\section{Modèle démographique et ses propriétés}

Le modèle démographique est défini par les équations de récurrence suivantes :

$$
\begin{aligned}
& \mathrm{x}_{1}(\mathrm{t}+1 \text { an })=\mathrm{s}_{0}\left[\mathrm{f}_{1} \mathrm{x}_{1}(\mathrm{t})+\mathrm{f}_{2} \mathrm{x}_{2}(\mathrm{t})+\mathrm{f}_{3} \mathrm{x}_{3}(\mathrm{t})\right] \\
& \mathrm{x}_{2}(\mathrm{t}+1 \text { an })=\mathrm{s}_{1} \mathrm{x}_{1}(\mathrm{t}) ; \\
& \mathrm{x}_{3}(\mathrm{t}+1 \text { an })=\mathrm{s}_{2} \mathrm{x}_{2}(\mathrm{t})+\mathrm{s}_{3} \mathrm{x}_{3}(\mathrm{t}) .
\end{aligned}
$$

Ces équations peuvent s'écrire sous la forme matricielle $\mathrm{x}(\mathrm{t}+1)=$ $\mathrm{A} x(\mathrm{t})$, où :

$$
\left[\begin{array}{l}
\mathrm{x}_{1}(\mathrm{t}+1 \text { an }) \\
\mathrm{x}_{2}(\mathrm{t}+1 \text { an }) \\
\mathrm{x}_{3}(\mathrm{t}+1 \text { an })
\end{array}\right]=\left[\begin{array}{ccc}
\mathrm{s}_{0} \mathrm{f}_{1} & \mathrm{~s}_{0} \mathrm{f}_{2} & \mathrm{~s}_{0} \mathrm{f}_{3} \\
\mathrm{~s}_{1} & 0 & 0 \\
0 & \mathrm{~s}_{2} & \mathrm{~s}_{3}
\end{array}\right]\left[\begin{array}{l}
\mathrm{x}_{1}(\mathrm{t}) \\
\mathrm{x}_{2}(\mathrm{t}) \\
\mathrm{x}_{3}(\mathrm{t})
\end{array}\right]
$$

La matrice A est appelée matrice de projection démographique ou matrice de Leslie (26). Lorsque les paramètres démographiques 
sont supposés constants d'année en année, la dynamique interannuelle est calculée par :

$\mathrm{x}(\mathrm{t}+\mathrm{T})=\mathrm{Ax}(\mathrm{T}-1)=\mathrm{A}^{2} \mathrm{x}(\mathrm{T}-2)=\ldots=\mathrm{A}^{\mathrm{T}} \mathrm{x}(\mathrm{t})$

A long terme et dans un environnement constant, le cheptel décrit par ce modèle converge vers un état stable : la croissance du cheptel devient exponentielle et le vecteur d'état $\mathrm{x}(\mathrm{t})$ converge vers une structure d'âge $\mathrm{v}$ (12). En d'autres termes, en régime stable, le cheptel croît chaque année avec le même taux $\lambda$, soit $\mathrm{x}(\mathrm{t}+1) \approx$ $\lambda \mathrm{x}(\mathrm{t})$, et la pyramide des âges reste constante d'année en année. La situation d'équilibre démographique stationnaire correspond au cas où $\lambda=1$. Le taux de multiplication annuel $\lambda$ et la structure d'âge stable v sont calculés respectivement par la valeur propre dominante de la matrice $\mathrm{A}$ et son vecteur propre à droite associé. Ils ne dépendent que de la matrice A et non du vecteur des effectifs décrivant l'état initial du cheptel.

\section{Calcul de la productivité}

La méthode proposée est décomposée en deux étapes principales :

- la première étape a consisté à estimer la fécondité et la probabilité de mort naturelle pour les différentes classes d'âge à partir des données disponibles et provenant en général de suivis de troupeaux. Ces paramètres déterminent le potentiel biologique du cheptel, notamment son potentiel de croissance démographique ;

- la seconde étape a consisté à déterminer, pour les différentes classes d'âge, les probabilités d'exploitation permettant d'engendrer un taux de croissance démographique fixé comme hypothèse pour le cheptel (en général, la situation d'équilibre stationnaire). Pour calculer ces probabilités d'exploitation, la méthode consiste à supposer qu'elles sont le produit d'une composante variable, notée $\varphi$, et d'une composante constante, appelée " structure d'exploitation de référence » et notée $\left\{\mathrm{P}_{\text {ref, } 0}(\exp ), \mathrm{P}_{\text {ref, }, 1}(\exp ), \mathrm{P}_{\text {ref }, 2}(\exp )\right.$, $\left.\mathrm{P}_{\text {ref }, 3}(\exp )\right\}$.

Dans l'exemple les probabilités de survie dans le troupeau ont été définies comme suit :

$$
\begin{aligned}
& \mathrm{s}_{0}=1-\mathrm{P}_{0, \text { ref }}(\text { mor })-\varphi \mathrm{P}_{0, \text { ref }}(\exp ) \\
& \mathrm{s}_{1}=1-\mathrm{P}_{1, \text { ref }}(\text { mor })-\varphi \mathrm{P}_{1, \text { ref }}(\exp ) \\
& \mathrm{s}_{2}=1-\mathrm{P}_{2, \text { ref }}(\text { mor })-\varphi \mathrm{P}_{2, \text { ref }}(\exp ) \\
& \mathrm{s}_{3}=1-\mathrm{P}_{3, \text { ref }}(\text { mor })-\varphi \mathrm{P}_{3, \text { ref }}(\exp )
\end{aligned}
$$

La composante $\varphi$ est un nombre représentant l'intensité globale d'exploitation. La structure d'exploitation de référence représente les pratiques de gestion des effectifs mises en œuvre par les éleveurs. Elle détermine l'importance respective des probabilités d'exploitation pour les différentes classes d'âge. La structure $\left\{\mathrm{P}_{\text {ref }, 0}(\exp ), \mathrm{P}_{\text {ref }, 1}(\exp ), \mathrm{P}_{\text {ref }, 2}(\exp ), \mathrm{P}_{\text {ref }, 3}(\exp )\right\}=\{1,1,1,1\}$ signifiait par exemple que toutes les classes d'âge sont exploitées au même taux. La structure $\left\{\mathrm{P}_{\text {ref }, 0}(\exp ), \mathrm{P}_{\text {ref, } 1}(\exp ), \mathrm{P}_{\text {ref, } 2}(\exp )\right.$, $\left.\mathrm{P}_{\text {ref }, 3}(\exp )\right\}=\{0,0,4,1\}$ signifie en revanche que seules les classes d'âge 2 et 3 sont exploitées, la classe 3 étant exploitée quatre fois plus que la classe 2. Cette structure d'exploitation de référence peut être calculée d'après les données disponibles, pour représenter les pratiques observées chez les éleveurs, ou bien fixée a priori, afin d'étudier l'effet de la structure d'exploitation sur la productivité du cheptel.

L'intensité d'exploitation $\varphi$ est utilisée pour contrôler le taux de croissance démographique du cheptel, par rapport à la structure d'exploitation de référence fixée. Lorsque $\varphi=0$, il n'y a pas d'exploitation, le cheptel croît. Plus $\varphi$ augmente, plus la croissance démographique diminue. La méthode consiste à calculer la valeur de $\varphi$ engendrant le taux de croissance démographique fixé comme objectif.
En pratique, les probabilités de survie $\mathrm{s}_{\mathrm{i}}$, fonctions décroissantes de l'intensité $\varphi$, sont placées dans la matrice de projection annuelle A. Le taux de multiplication annuel $\lambda$ est lui-même une fonction décroissante de $\varphi$ (les fécondités, les probabilités de mort naturelle et la structure d'exploitation de référence sont supposées fixées dans le modèle). Il s'agit de calculer la valeur $\varphi_{\text {objectif }}$ de $\varphi$ assurant la condition $\lambda=\lambda_{\text {objectif }}\left(\lambda_{\text {objectif }}=1\right.$ pour l'équilibre stationnaire $)$.

Une fois cette valeur calculée, les effectifs des femelles exploitables dans l'année sont déduits en fixant comme vecteur d'état initial du cheptel $\mathrm{x}(\mathrm{t})$ un vecteur proportionnel à la structure d'âge stable du cheptel exploité, c'est-à-dire $\mathrm{x}(\mathrm{t})=\mathrm{n} \mathrm{v}$ où $\mathrm{n}$ représente l'effectif total du cheptel et $\mathrm{v}=\left\{\mathrm{v}_{1}, \mathrm{v}_{2}, \mathrm{v}_{3}\right\}$ est le vecteur propre dominant standardisé $\left(\mathrm{v}_{1}+\mathrm{v}_{2}+\mathrm{v}_{3}=1\right)$ de la matrice de projection annuelle $\mathrm{A}$ (de manière générale, $\mathrm{v}$ vérifie $\mathrm{A} \mathrm{v}=\lambda \mathrm{v}$; à l'équilibre stationnaire, $v$ vérifie $A v=v$ ). Les effectifs des femelles exploitables dans l'année sont définis comme suit :

- pour la classe $0, z_{0}=n \varphi_{\text {objectif }} P_{0 \text {,ref }}(\exp )\left(f_{1} v_{1}+f_{2} v_{2}+f_{3} v_{3}\right)$;

- pour la classe $1, \mathrm{z}_{1}=\mathrm{n} \varphi_{\text {objectif }} \mathrm{P}_{1, \text { ref }}(\exp ) \mathrm{v}_{1}$;

- pour la classe $2, z_{2}=n \varphi_{\text {objectif }} P_{2, \text { ref }}(\exp ) v_{2}$;

- pour la classe $3, z_{3}=n \varphi_{\text {objectif }} P_{3, \text { ref }}(\exp ) v_{3}$.

La productivité du cheptel d'effectif total $n$, notée $\mathrm{W}$, est définie comme la somme pondérée des effectifs des femelles exploitables dans l'année : $\mathrm{W}=\mathrm{c}_{0} \mathrm{z}_{0}+\mathrm{c}_{1} \mathrm{z}_{1}+\mathrm{c}_{2} \mathrm{z}_{2}+\mathrm{c}_{3} \mathrm{z}_{3}$, où $\mathrm{c}_{\mathrm{i}}$ représente un paramètre de pondération, comme le poids ou le prix de vente des animaux. $\mathrm{Si}_{\mathrm{i}}=1, \mathrm{~W}$ représente la productivité numérique $\mathrm{du}$ cheptel.

Pour la réalisation des calculs, les équations précédentes sont exprimées sous forme matricielle et la valeur $\varphi_{\text {objectif }}$ est calculée à l'aide de la méthode itérative de Newton-Raphson (28).

\section{Exemple numérique}

En prenant les valeurs suivantes comme paramètres d'entrée pour le modèle :

- fécondités $\left\{\mathrm{f}_{1}, \mathrm{f}_{2}, \mathrm{f}_{3}\right\}=\{0 ; 0,45 ; 0,45\}$;

- mortalités naturelles $\left\{\mathrm{P}_{0}\right.$ (mor), $\mathrm{P}_{1}$ (mor), $\mathrm{P}_{2}$ (mor), $\mathrm{P}_{3}$ (mor) $\}=$ $\{0,10 ; 0,10 ; 0,10 ; 0,05\}$;

- structure d'exploitation de référence $\left\{\mathrm{P}_{\text {ref }, 0}(\exp ), \mathrm{P}_{\text {ref }, 1}(\exp )\right.$, $\left.\mathrm{P}_{\text {ref }, 2}(\exp ), \mathrm{P}_{\text {ref }, 3}(\exp )\right\}=\{0 ; 0 ; 4 ; 1\}$;

nous avons obtenu en sorties du modèle :

- l'intensité d'exploitation engendrant l'équilibre stationnaire $\varphi_{\text {objectif }}=0,141$;

- la structure d'âge stable $\left\{\mathrm{v}_{1}, \mathrm{v}_{2}, \mathrm{v}_{3}\right\}=\{0,29 ; 0,26 ; 0,45\}$;

- la productivité numérique $\mathrm{W}=210$ femelles, pour un cheptel d'effectif total $\mathrm{n}=1000$ femelles.

Cette productivité numérique, mesure du potentiel productif du cheptel d'effectif total $\mathrm{n}$, a été calculée par rapport aux hypothèses fixées, c'est-à-dire à la structure d'exploitation de référence et au taux de multiplication annuel fixés comme objectif. Si l'une de ces hypothèses varie, l'intensité d'exploitation $\varphi_{\text {objectif }}$ et la productivité $\mathrm{W}$ varient également pour un même potentiel biologique de départ, c'est-à-dire pour les mêmes fécondités et probabilités de mort naturelle. Ainsi, lorsque plusieurs cheptels sont comparés, leur productivité doit être calculée en fixant une structure d'exploitation et une hypothèse de croissance démographique communes à l'ensemble des cheptels.

\section{Extension au cas d'une étude pluriannuelle}

L'exemple précédent n'a concerné qu'une année de production. La méthode s'applique aussi quand la productivité est évaluée sur une période pluriannuelle. Par exemple, pour une période de trois 
années, la matrice de projection est $A=A_{t+2} A_{t+1} A_{t}$, où $A_{t}$ représente la matrice de projection pour l'année [t, $t+1$ an]. La valeur propre dominante $\lambda$ de A représente le taux de multiplication pluriannuel de la population (le taux annuel moyen a été $\lambda^{1 / 3}$ ). Le calcul de la productivité pluriannuelle s'effectue selon le même principe que le précédent :

- la première étape est de fixer une structure d'exploitation de référence pour les trois années. Cette structure peut être identique pour les trois matrices annuelles ou varier d'une année sur l'autre ;

- la seconde étape consiste à rechercher l'intensité d'exploitation $\varphi_{\text {objectif }}$ qui engendre une valeur propre $\lambda$ de la matrice A égale au taux de multiplication pluriannuel fixé comme objectif. La productivité $\mathrm{W}$ correspond à la somme des productions observées sur les trois années pour l'intensité d'exploitation $\varphi_{\text {objectif. }}$ Dans le cas d'une productivité monétaire, les valeurs peuvent être actualisées chaque année.

\section{Cas du modèle démographique par quinzaine}

Dans le modèle proposé, le vecteur d'état de la population est calculé de quinzaine en quinzaine d'après l'équation de récurrence $(27,29)$ :

$\mathrm{x}(\mathrm{k}+1$ quinzaine $)=\mathrm{B}_{\mathrm{k}} \mathrm{x}(\mathrm{k})$

Le vecteur d'état $\mathrm{x}(\mathrm{k})$ contient les effectifs des femelles et des mâles présents en début de quinzaine $\mathrm{k}$ dans les différentes classes d'âge (la taille des classes d'âge a été de $15 \mathrm{j}$ ). Chaque matrice $B_{k}$ a la structure d'une matrice de Leslie conventionnelle (figure 1) et représentait conjointement le cycle de vie des femelles et celui des mâles. Sur une échelle annuelle (24 quinzaines), la dynamique a été décrite par l'équation :

$\mathrm{x}(\mathrm{t}+1$ an $)=\mathrm{B}_{24} \ldots \mathrm{B}_{1} \mathrm{x}(\mathrm{t})=\mathrm{A} \mathrm{x}(\mathrm{t})$

A long terme, le modèle a les mêmes propriétés de convergence que le modèle de Leslie annuel, mais la matrice de projection A est plus complexe. Le taux de multiplication asymptotique annuel $\lambda$ et la structure stable $\mathrm{v}$ sont calculés par la valeur propre dominante de la matrice $\mathrm{A}$ et son vecteur propre à droite associé. Le taux $\lambda$ ne dépend que des paramètres démographiques des femelles, le modèle est appelé femelle-dominant. Les paramètres des mâles affectent uniquement le rapport femelles/mâles et la structure d'âge des mâles dans le cheptel.
Pour le calcul de la productivité, les extensions par rapport à la méthode décrite dans l'exemple du modèle à pas de temps annuel ont été les suivantes $(28,29)$ :

- la prise en compte des mâles ;

- la prise en compte des importations d'animaux ;

- la définition d'une structure d'exploitation nette de référence, représentant l'importance respective des niveaux d'exploitations nettes (balance des exploitations et importations) pour les différents sexes, classes d'âge et quinzaines dans l'année.

Pour un sexe, une classe d'âge et une quinzaine donnés, la « probabilité » d'exploitation nette a été $\mathrm{P}(\exp )$ - $\mathrm{P}(\mathrm{imp})$, où $\mathrm{P}(\mathrm{imp})$ représente le paramètre d'importation des animaux dans les troupeaux. A la différence de $\mathrm{P}(\exp ), \mathrm{P}(\mathrm{imp})$ n'est pas une probabilité au sens strict car une partie des animaux importés dans les troupeaux peuvent provenir d'une population différente de celle des cheptels étudiés. Le modèle suppose que l'effectif des animaux importés dans les troupeaux durant une quinzaine est proportionnel, avec un coefficient $\mathrm{P}(\mathrm{imp})$, à l'effectif des animaux présents au début de la quinzaine :

Nombre d'animaux importés $=\mathrm{P}(\mathrm{imp}) \mathrm{x}$ nombre d'animaux présents

Cette hypothèse se justifie si les troupeaux échantillonnés pour l'estimation des paramètres démographiques ont un effectif qui reste proportionnel à celui de l'ensemble du cheptel (27). Dans le cas d'un pas de temps court comme la quinzaine, le paramètre $\mathrm{P}$ (imp) présente des valeurs toujours très inférieures à 1 et peut être traité (en première approximation) comme une probabilité (28). Dans la suite du texte, par abus de langage, les paramètres $\mathrm{P}(\exp )$ - P(imp) (qui peuvent être négatifs) et P(imp) ont été appelés respectivement probabilité d'exploitation nette et probabilité d'importation.

Pour contrôler le taux de croissance démographique du cheptel, la méthode suppose que les probabilités d'exploitation varient indépendamment des probabilités d'importation : ces dernières ne dépendent pas du nombre $\varphi$ qui contrôle l'intensité globale d'exploitation. Par ailleurs, pour respecter l'hypothèse de femelledominance, $\varphi$ ne contrôle pas l'exploitation des mâles (le taux de multiplication annuel $\lambda$ ne dépend que des paramètres des femelles). Les probabilités de survie pour une classe d'âge i et une quinzaine $\mathrm{k}$ données sont alors définies comme suit :

$x(k)=\left[x_{f, 1}(k), \ldots, x_{f, J}(k), x_{m, 1}(k), \ldots, x_{m, L}(k)\right]^{\prime}$

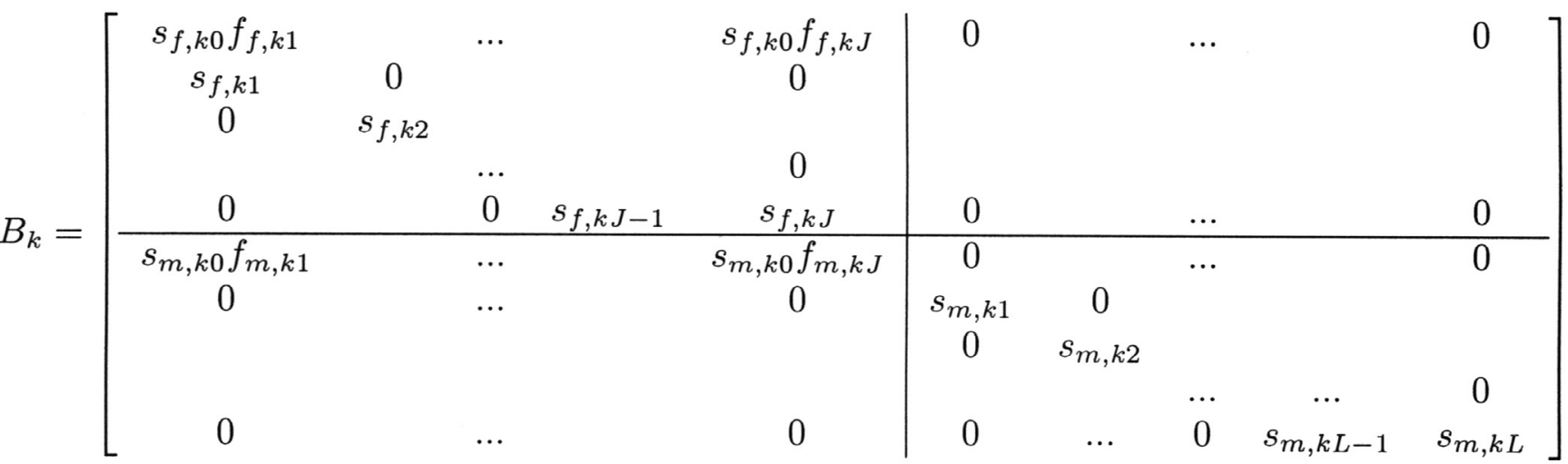

Figure 1 : structures du vecteur d'état $x(k)$ du cheptel et de la matrice de projection $B_{k}$ pour la quinzaine $k$. Les paramètres $f$ et $s$ représentent respectivement les fécondités et les probabilités de survie dans le troupeau pour les différentes classes d'âge (dans la matrice représentée, il y a J classes d'âge femelles et L classes d'âge mâles). 
- pour les femelles par $s_{f, k i}=1-P_{f, k i}($ mor $)-\varphi P_{\text {ref,f,ki }}(\exp )+$ $\mathrm{P}_{\text {ref,f,ki }}(\mathrm{imp})$;

- pour les mâles par $\mathrm{s}_{\mathrm{m}, \mathrm{ki}}=1-\mathrm{P}_{\mathrm{m}, \mathrm{ki}}(\mathrm{mor})-\mathrm{P}_{\mathrm{ref}, \mathrm{m}, \mathrm{ki}}(\exp )+$ $\mathrm{P}_{\text {ref,m,ki }}$ (imp).

Le principe du calcul de la productivité d'un cheptel est identique à celui présenté dans l'exemple du modèle à pas de temps annuel. Les paramètres de fécondité, de mort naturelle, d'exploitation et d'importation sont placés dans les matrices de projection par quinzaine $B_{k}$ dont le produit a engendré la matrice de projection annuelle $\mathrm{A}$. Le taux de multiplication annuel à long terme $\lambda$, valeur propre dominante de la matrice $\mathrm{A}$, est une fonction décroissante de l'intensité $\varphi$. La valeur $\varphi_{\text {objectif }}$ permettant d'atteindre le taux de multiplication annuel fixé comme objectif $\left(\lambda_{\text {objectif }}\right)$ est calculée de manière itérative (28). Pour un stade d'âge i et une quinzaine $\mathrm{k}$, l'effectif net des animaux exploités est calculé comme suit :

- pour les femelles par $\mathrm{z}_{\mathrm{f}, \mathrm{ki}}=\left[\varphi_{\text {objectif }} \mathrm{P}_{\text {ref,f,ki }}(\exp )-\mathrm{P}_{\text {ref,f,ki }}(\mathrm{imp})\right]$ $\mathrm{x}_{\mathrm{f}, \mathrm{i}}(\mathrm{k})$;

- pour les mâles par $\mathrm{z}_{\mathrm{m}, \mathrm{ki}}=\left[\mathrm{P}_{\mathrm{ref}, \mathrm{m}, \mathrm{ki}}(\exp )-\mathrm{P}_{\mathrm{ref}, \mathrm{m}, \mathrm{ki}}(\mathrm{imp})\right] \mathrm{x}_{\mathrm{m}, \mathrm{i}}(\mathrm{k})$.

Le vecteur représentant le cheptel en début d'année est déterminé par $\mathrm{x}(\mathrm{t})=\mathrm{n} \mathrm{v}$. La somme des effectifs des animaux exploités (nets) sur l'ensemble des classes d'âge et des quinzaines dans l'année, somme éventuellement pondérée par des poids vifs ou des prix de vente, donne la productivité W. La productivité totale peut être décomposée en une productivité femelle et une productivité mâle : $\mathrm{W}=\mathrm{W}_{\mathrm{f}}+\mathrm{W}_{\mathrm{m}}$.

\section{Calcul d'intervalles de confiance pour les productivités estimées}

En pratique, les paramètres démographiques sont estimés à l'aide de régressions ajustées sur un échantillon observé. Ils sont connus avec une certaine imprécision (écart-type). La productivité W, fonction de ces paramètres, est par conséquent estimée avec une certaine incertitude qui doit être prise en compte dans les analyses comparatives entre cheptels. Les estimations de W doivent être accompagnées d'intervalles de confiance, permettant d'encadrer les vraies valeurs (inconnues), avec un certain niveau de confiance. Les comparaisons entre cheptels doivent s'appuyer sur des tests statistiques pour décider si les différences observées sont significatives ou si elles peuvent provenir de l'incertitude due à l'échantillonnage.

Ce problème est ignoré dans la littérature zootechnique concernant les indicateurs démographiques de la productivité des cheptels. A la connaissance des auteurs, seuls Ankers et coll. (2) l'ont abordé dans le cadre des modèles de production à l'équilibre. Ces auteurs ont évalué l'effet d'un traitement prophylactique sur la productivité des ovins en Gambie. L'étude a porté sur quatre villages et chaque village comprenait un lot d'animaux traités et un lot d'animaux témoins. Les productivités des lots d'animaux traités et témoins ont été estimées séparément pour chaque village et les moyennes des productivités des lots traités et témoins ont été comparées par un test de Student. Cette procédure n'a pas tenu compte des variances d'échantillonnage des paramètres démographiques estimés pour chaque lot d'animaux.

Pour calculer les intervalles de confiance des estimations de W et réaliser des tests statistiques, il est nécessaire de calculer la loi de probabilité des estimateurs (ou au moins leur variance). La productivité $\mathrm{W}$ étant une fonction complexe des paramètres démographiques, le calcul analytique de cette loi de probabilité pose des problèmes importants.

Une autre possibilité est d'utiliser une procédure de ré-échantillonnage (bootstrap non paramétrique ; 15), mise en oeuvre par exemple en écologie pour l'étude de la dynamique des populations sauvages $(9,22,33)$. Nous avons adapté cette technique au rééchantillonnage des carrières des animaux présents dans l'échantillon pour prendre en compte les corrélations existant entre les différents paramètres démographiques $(28,29)$.

La carrière d'une femelle a été résumée par son entrée dans l'un des troupeaux du cheptel (naissance ou importation), son devenir (survie dans le troupeau, mort naturelle ou exploitation), l'ensemble de ses mises bas et le nombre des produits obtenus lors de chaque mise bas observée. La carrière d'un mâle a été résumée par son entrée dans l'un des troupeaux du cheptel et son devenir. La procédure de ré-échantillonnage a consisté à construire un nombre de $\mathrm{B}$ échantillons bootstrap à partir de l'échantillon d'origine des carrières animales. Chaque échantillon de même taille que l'original ( $\mathrm{N}$ carrières) a été construit en réalisant un échantillonnage aléatoire avec remises et à probabilités égales $(1 / \mathrm{N})$ des carrières dans l'échantillon d'origine. Les fécondités et les probabilités de mort naturelle ont été estimées pour chaque échantillon à partir des mêmes modèles de régression que ceux utilisés pour l'échantillon d'origine, puis ont été placées dans le modèle démographique pour calculer les productivités $\mathrm{W}_{(\mathrm{b})}$, avec $\mathrm{b}$ variant de 1 à $\mathrm{B}$ (la même structure d'exploitation nette de référence a été conservée pour tous les échantillons). Un nombre suffisamment grand d'échantillons bootstrap (au moins 1000 , voire 10000 en pratique) a permis d'estimer la distribution de la productivité estimée $\mathrm{W}$ (ou de toute fonction de cette productivité : différences ou rapports de productivités calculés pour différents cheptels) à partir de la distribution observée des productivités $\mathrm{W}_{(\mathrm{b})}$.

Lorsque cette distribution se rapproche de celle d'une loi normale, l'intervalle de confiance au risque $\alpha$ de la productivité peut être estimé par $\mathrm{W} \pm \mathrm{z}_{1-\alpha / 2} \mathrm{se}_{\mathrm{B}}$, où $\mathrm{z}_{1-\alpha / 2}$ est le quantile d'ordre 1- $\alpha / 2 \mathrm{de}$ la loi normale centrée réduite $\left[\mathrm{P}\left(\mathrm{Z}>\mathrm{z}_{1-\alpha / 2}\right)=\alpha / 2\right.$; par exemple, si $\alpha=0,05$, alors $\left.\mathrm{Z}_{1-\alpha / 2}=1,96\right]$ et $\mathrm{se}_{\mathrm{B}}$ l'écart-type estimé de $\mathrm{W}$ calculé d'après les productivités $\mathrm{W}_{(\mathrm{b})}$ :

$$
\mathrm{se}_{\mathrm{B}}=\sqrt{\frac{\sum_{\mathrm{b}=1}^{\mathrm{B}}\left[\mathrm{w}_{(\mathrm{b})}-\left(\sum_{\mathrm{b}=1}^{\mathrm{B}} \frac{\mathrm{w}_{(b)}}{\mathrm{B}}\right)\right]^{2}}{B-1}}
$$

Dans le cas contraire, la méthode des percentiles ou d'autres méthodes plus élaborées sont préférables $(12,15)$.

\section{APPLICATION DU MODELE DEMOGRAPHIQUE PAR QUINZAINE}

Le modèle matriciel périodique a été utilisé pour évaluer l'efficacité d'un plan de prophylaxie sur la productivité d'un cheptel sédentaire d'ovins domestiques dans la région de Kolda au Sénégal. Cette région subhumide est caractérisée par une saison des pluies de cinq mois (juillet-novembre), suivie d'une saison sèche de sept mois. Les paysans sont des Peuls sédentarisés pratiquant l'agriculture (riz, arachide, maïs, coton...) et l'élevage (bovins et petits ruminants). Les ovins sont de race Djallonké de petit format et trypanotolérante, élevés uniquement pour la viande et la vente d'animaux sur pieds. Ils sont élevés sur parcours naturels selon un mode de conduite très extensif (16).

L'estimation des paramètres démographiques du cheptel et les calculs démographiques ont été réalisés respectivement avec les logiciels Splus 2000 (32) et Matlab 5.3 (42). 


\section{Données}

Les données sont provenues d'un suivi d'élevages réalisé dans le cadre du programme Pathologie et productivité des petits ruminants (44). Au cours de ce programme, les troupeaux suivis ont été sélectionnés sur des critères de représentativité agro-écologique, d'accessibilité en toute saison et de volontariat des éleveurs. Dans chaque troupeau, tous les animaux ont été identifiés. Des enquêteurs ont effectué des visites bimensuelles au cours desquelles les événements démographiques ont été notés (naissances, morts, entrées et sorties du troupeau) selon un protocole précis (18). Les données ont été saisies et validées au cours des travaux de terrain. Plus récemment, elles ont été placées dans une base de données relationnelle pour faciliter leur traitement (24).

Ces données ont été relevées lors des exercices agricoles de 1985 , 1986 et 1987 (périodes allant du $1^{\text {er }}$ juillet de l'année calendaire au 30 juin de l'année suivante). L'un des objectifs du suivi a été d'estimer l'effet de mesures de prévention de la pasteurellose respiratoire (vaccination) et du parasitisme gastro-intestinal (vermifugation) au cours de ces périodes $(19,43)$.

Pour chaque exercice, la vaccination a consisté en deux injections d'un vaccin inactivé contre la pasteurellose respiratoire (Pasteurella multocida types A et D, Pasteurellad ${ }^{\mathrm{ND}}$, Isra-Lnerv, Dakar, Sénégal). Le facteur décrivant la vaccination a été codé avec la lettre $\mathrm{V}$ (vacciné) ou $\mathrm{C}$ (témoin). La vermifugation a été réalisée en saison des pluies, à l'aide de fenbendazole (Panacur ${ }^{\mathrm{ND}}$, Hoechst, 7,5 mg/kg PV, deux administrations) lors de l'exercice 1985, puis de morantel (Exhelm ${ }^{\mathrm{ND}}$, Pfizer, $10 \mathrm{mg} / \mathrm{kg} \mathrm{PV}$, trois administrations lors des exercices de 1986 et 1987). Le facteur décrivant la vermifugation a été codé avec la lettre $\mathrm{D}$ (vermifugé) ou C (témoin).

Les villages ont été attribués aléatoirement à l'un des quatre groupes de traitement. Tous les animaux d'un même village ont reçu le même traitement. Les mêmes villages ont été suivis sur les trois exercices. Les quatre groupes de troupeaux ont été notés de la manière suivante :

- CC (non vaccinés et non vermifugés);

- CD (non vaccinés et vermifugés);

- VC (vaccinés et non vermifugés);

- VD (vaccinés et vermifugés).
Les effectifs des troupeaux et des animaux suivis dans les différents groupes sont présentés dans le tableau I.

Dans cette étude, deux analyses séparées ont été effectuées : l'analyse de l'exercice 1987 seul (plan 1987), puis l'analyse globale des trois exercices (plan 1985-1987).

\section{Estimation des paramètres démographiques et des productivités}

Pour chaque plan, les paramètres démographiques des matrices de projection par quinzaine $B_{k}$ ont été estimés d'après des modèles statistiques de régression (modèles linéaires généralisés avec des liens « log-log complémentaire » et « logit»). Les facteurs suivants ont été introduits dans les modèles : (1) vaccination, (2) vermifugation, (3) exercice (pour le plan 1985-1987), (4) mois de l'année, (5) sexe et (6) groupe d'âge (0-3 mois, 3-6 mois, 6-12 mois, 1-2 ans, $>2$ ans).

Pour chaque paramètre démographique, une procédure a été utilisée pour sélectionner le meilleur modèle de régression au sens du critère d'information d'Akaike (AIC ; 11). La sélection d'un modèle selon l'AIC obéit au principe de parcimonie, consistant à conserver les paramètres des modèles de régression expliquant la structure significative des données et à exclure les paramètres additionnels que ne peuvent justifier les données disponibles. Ce principe revient à trouver un compromis entre le biais et la variance d'échantillonnage des estimateurs.

La structure d'exploitation nette de référence a été calculée par la moyenne arithmétique des structures des quatre groupes de troupeaux (CC, VC, CD, VD) et, dans le plan 1985-1987, pour les trois exercices (la même structure de référence a été fixée pour les trois exercices). La structure utilisée pour le plan 1987 est présentée aux figures 2 et 3 . La structure d'exploitation des mâles a été caractérisée par un fort effet de la fête de la Tabaski (sacrifice des moutons lors de la fête musulmane de l'Aïd El Kébir) en août, et l'augmentation rapide du niveau d'exploitation avec l'âge (figure 2). Les femelles ont présenté une structure d'exploitation plus complexe, avec un effet Tabaski moins net (figure 3).

La productivité numérique de chaque groupe de troupeaux a été estimée en situation d'équilibre démographique stationnaire pour un effectif total théorique de $n=1000$ animaux. Les effets

\section{Tableau I}

Effectifs des troupeaux et des ovins par groupe de traitement durant les exercices agricoles de 1985, 1986 et 1987 à Kolda, Sénégal

\begin{tabular}{|c|c|c|c|c|c|c|c|}
\hline \multirow[t]{3}{*}{ Exercices } & \multirow[t]{3}{*}{ Vaccination } & \multicolumn{2}{|c|}{ Troupeaux } & \multicolumn{2}{|c|}{ Femelles } & \multicolumn{2}{|c|}{ Mâles } \\
\hline & & \multicolumn{2}{|c|}{ Vermifugation } & \multicolumn{2}{|c|}{ Vermifugation } & \multicolumn{2}{|c|}{ Vermifugation } \\
\hline & & C & D & C & D & C & D \\
\hline \multirow[t]{2}{*}{1985} & $\mathrm{C}$ & 16 & 27 & 160 & 254 & 87 & 185 \\
\hline & V & 27 & 23 & 287 & 240 & 152 & 168 \\
\hline \multirow[t]{2}{*}{1986} & $\mathrm{C}$ & 15 & 24 & 177 & 297 & 96 & 200 \\
\hline & V & 23 & 24 & 265 & 270 & 147 & 173 \\
\hline \multirow[t]{2}{*}{1987} & C & 13 & 23 & 171 & 358 & 116 & 228 \\
\hline & V & 15 & 23 & 156 & 277 & 75 & 174 \\
\hline
\end{tabular}

$\mathrm{CC}$ : non vacciné et non vermifugé ; $\mathrm{CD}$ : non vacciné et vermifugé ; $\mathrm{VC}$ : vacciné et non vermifugé ; VD : vacciné et vermifugé 


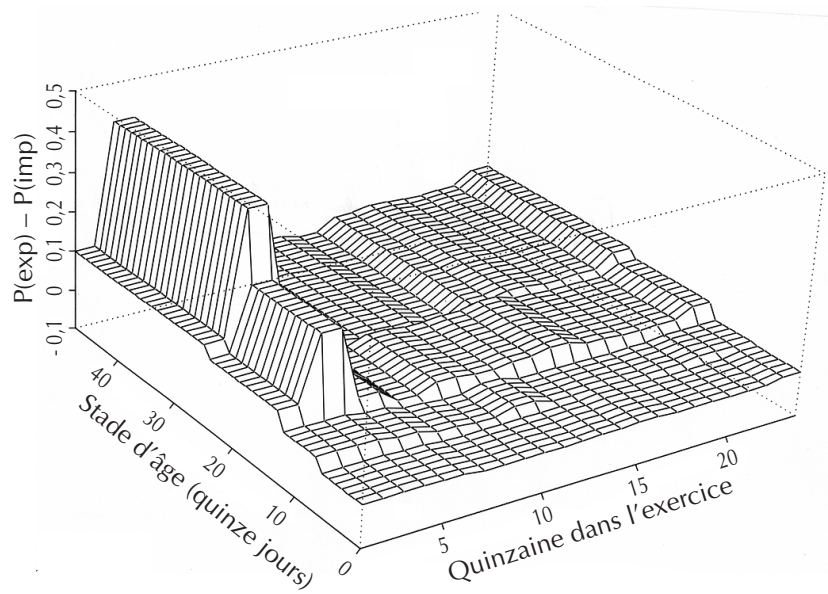

Figure 2 : structure d'exploitation nette $P(\exp )-P($ imp $)$, par classe d'âge de 15 j et par quinzaine des mâles de moins de deux ans, utilisée pour évaluer l'effet du plan de prophylaxie de 1987 sur la productivité des ovins Djallonké de Kolda (Sénégal). Cette structure a été estimée d'après les données de 1987. Les mâles survivants ont été supposés tous réformés à deux ans. Les classes d'âge de $15 j$ vont de 0 à 48 (de 0 à 2 ans) et les quinzaines vont de 1 (première quinzaine de juillet) à 24 (dernière quinzaine de juin). En 1987, la fête de la Tabaski a eu lieu en août (quinzaine $\left.n^{\circ} 3\right)$.

vaccination et vermifugation ont été estimés respectivement par $\left(\mathrm{W}_{\mathrm{VC}}+\mathrm{W}_{\mathrm{VD}}\right)-\left(\mathrm{W}_{\mathrm{CC}}+\mathrm{W}_{\mathrm{CD}}\right)$ et $\left(\mathrm{W}_{\mathrm{CD}}+\mathrm{W}_{\mathrm{VD}}\right)-\left(\mathrm{W}_{\mathrm{CC}}+\mathrm{W}_{\mathrm{VC}}\right)$. $\mathrm{L}$ 'interaction vaccination-vermifugation a été estimée par $\left(\mathrm{W}_{\mathrm{VD}}-\right.$ $\left.\mathrm{W}_{\mathrm{VC}}\right)-\left(\mathrm{W}_{\mathrm{CD}}-\mathrm{W}_{\mathrm{CC}}\right)$.

Le calcul des intervalles de confiance n'a été effectué que pour le plan 1987. Les lois de probabilité des indicateurs de productivité ont été estimées à partir de $\mathrm{B}=1000$ réplications bootstrap. La taille de l'échantillon d'origine a été de $\mathrm{N}=1555$ carrières animales. Les intervalles de confiance des effets et de l'interaction ont été calculés pour un risque de 5 p. 100.

\section{Synthèse des résultats}

\section{Plan 1987}

Des résultats déjà publiés (29) sont repris ici. La vaccination et l'interaction vaccination-vermifugation n'ont pas présenté d'effet significatif sur la productivité numérique (figure 4). En revanche, la vermifugation a présenté un effet positif significatif. Le gain de productivité numérique dû à la vermifugation a été quantifié par le rapport :

$$
\mathrm{R}_{\text {num }}=\frac{\mathrm{W}_{\mathrm{CD}}+\mathrm{W}_{\mathrm{VD}}}{\mathrm{W}_{\mathrm{CC}}+\mathrm{W}_{\mathrm{VC}}}
$$

où $\mathrm{W}$ représente la productivité numérique totale. Le rapport bénéfice/coût de la vermifugation a été calculé par :

$$
\mathrm{R}_{\mathrm{BC}}=\frac{\left(\mathrm{W}_{\mathrm{CD}}+\mathrm{W}_{\mathrm{VD}}\right)+\left(\mathrm{W}_{\mathrm{CC}}+\mathrm{W}_{\mathrm{VC}}\right)}{\mathrm{C}_{\mathrm{CD}}+\mathrm{C}_{\mathrm{VD}}}
$$

où $\mathrm{W}$ représente la productivité monétaire totale et $\mathrm{C}$ le coût lié à la vermifugation. Les productivités monétaires ont été calculées en multipliant les effectifs des animaux exploités (nets) par sexe, classe d'âge et quinzaine par les prix de vente des animaux des catégories considérées. Les prix de vente ont été estimés d'après les prix observés lors de l'exercice de 1996 dans la région de Kolda, plus représentatifs de la période actuelle que ceux de l'exercice de 1987. Deux périodes ont été considérées dans

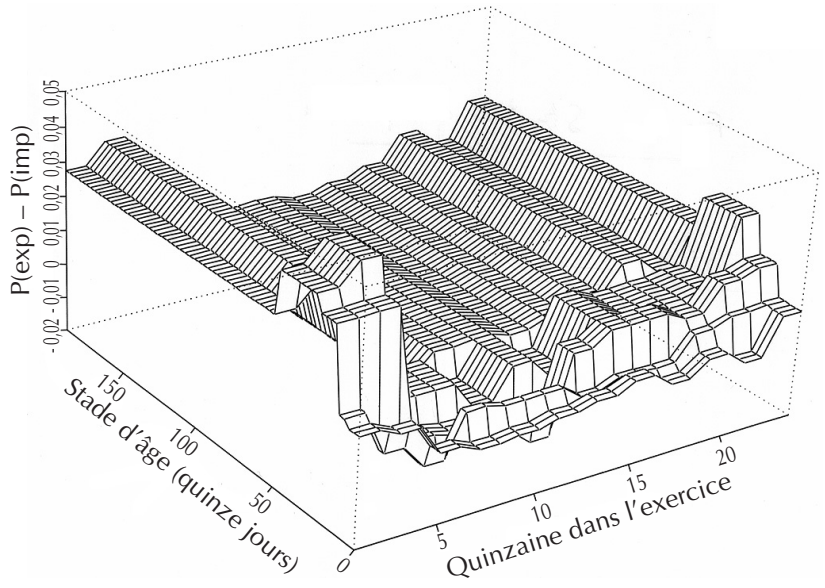

Figure 3 : structure d'exploitation nette des femelles P(exp) $P($ imp), par classe d'âge de $15 j$ et quinzaine, utilisée pour évaluer l'effet du plan de prophylaxie de 1987 sur la productivité des ovins Djallonké de Kolda (Sénégal). Cette structure a été estimée d'après les données observées en 1987. Les classes d'âge de $15 j$ vont de 0 à 193 (de 0 à 8 ans et plus) et les quinzaines vont de 1 (première quinzaine de juillet) à 24 (dernière quinzaine de juin). En 1987, la fête de la Tabaski a eu lieu en août (quinzaine $n^{\circ} 3$ ).
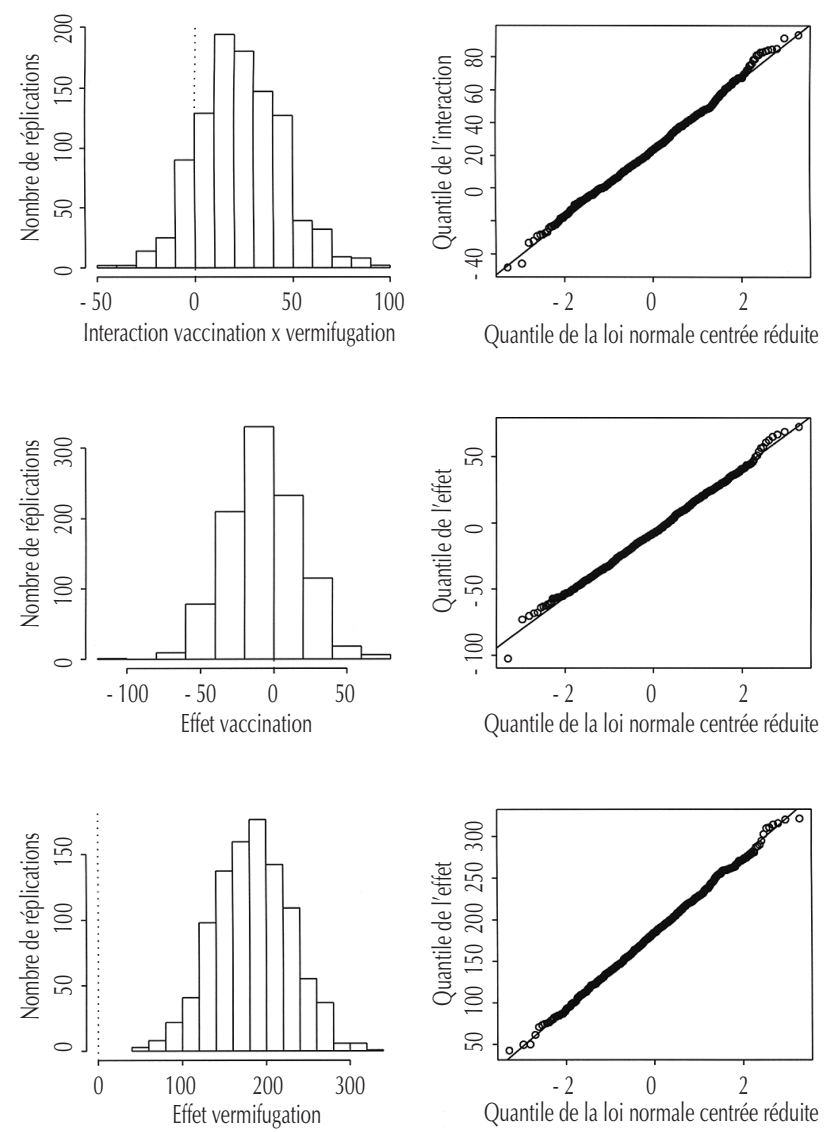

Figure 4 : quantiles des lois de probabilité empiriques calculés d'après 1000 réplications bootstrap de la productivité numérique totale (femelles + mâles) ; interaction $\left(W_{Y D}-\right.$ $\left.W_{V C}\right)-\left(W_{C D}-W_{C C}\right)$, effet vaccination $\left(W_{V C}+W_{Y D}\right)-\left(W_{C C}\right.$ $\left.+W_{C D}\right)$ et effet vermifugation $\left(W_{C D}+W_{V D}\right)-\left(W_{C C}+W_{V C}\right)$ (l'abscisse des trois histogrammes représente la valeur de l'interaction et des effets en effectif d'animaux exploitables). Les quantiles des lois empiriques ont été représentés en fonction des quantiles de la loi normale centrée réduite. L'alignement des points le long d'une droite montre que les effets et l'interaction suivent approximativement une loi normale. 
l'exercice agricole : la période de la fête de la Tabaski et la période hors de la fête de la Tabaski (tableau II). Le coût de vermifugation a été calculé en multipliant le prix d'un traitement (prix d'achat d'une dose de produit) par l'effectif des animaux d'âge supérieur à trois mois présents aux deux dates d'administration.

Les productivités numériques (femelles, mâles et totales) sont présentées dans le tableau III. Le rapport des productivités numériques totales a été estimé à $\mathrm{R}_{\text {num }}=1,22$, ce qui représente un gain de 91 animaux produits pour une population de $n=1000$ ovins, avec un intervalle de confiance de $[1,10 ; 1,36]$. Le rapport bénéfice/coût a été estimé à $\mathrm{R}_{\mathrm{BC}}=3,70$ avec un intervalle de confiance de $[1,88 ; 5,39]$ (29 pour une présentation détaillée), autrement dit pour 1 Fcfa investi pour vermifuger les animaux, le gain moyen attendu en productivité financière a été de $3,70 \mathrm{Fcfa}$.

\section{Plan 1985-1987}

Le diagnostic zootechnique a été semblable à celui obtenu pour le plan 1987. Les rapports entre les productivités numériques totales des groupes vaccinés et non vaccinés ont tous été inférieurs à 1 (tableau IV). Pour la vermifugation, le rapport $\left(\mathrm{W}_{\mathrm{CD}}+\mathrm{W}_{\mathrm{VD}}\right) /$ $\left(\mathrm{W}_{\mathrm{CC}}+\mathrm{W}_{\mathrm{VC}}\right)$ a été de 1,272 , montrant un gain de productivité après vermifugation légèrement supérieur à celui observé pour le plan $1987(1,223)$, résultant des fortes mortalités dans le groupe VC en 1986 et 1987, difficilement imputables à la vaccination.

Une analyse de la sensibilité des résultats à l'hypothèse d'équilibre démographique stationnaire a été effectuée en calculant le rapport des productivités numériques $\left(\mathrm{W}_{\mathrm{CD}}+\mathrm{W}_{\mathrm{VD}}\right) /\left(\mathrm{W}_{\mathrm{CC}}+\mathrm{W}_{\mathrm{VC}}\right)$ pour différentes valeurs du taux de multiplication fixé comme objectif $\left(\lambda_{\text {objectif }}\right)$. Ce rapport est passé de 1,162 à 1,481 pour des valeurs du taux de multiplication annuel $\left(\lambda_{\text {objectif }}{ }^{1 / 3}\right)$ respectivement de 0,8 et 1,2 .

\section{DISCUSSION}

\section{Effet des prophylaxies}

Les résultats sur l'efficacité de la vermifugation concordent avec ceux de Tillard (43) pour la région de Kolda. Les productivités numériques et monétaires calculées en 1987 ont montré un effet positif significatif de la vermifugation. Pour la productivité numérique, cet effet a été confirmé par les résultats obtenus entre 1985 et 1987 (sous réserve des tests de significativité non effectués). Dans son étude, Tillard considère une intervention prophylactique planifiée sur une période de cinq exercices agricoles successifs. Les données utilisées pour estimer la fécondité et la mortalité des animaux vermifugés et non vermifugés englobent les jeux de données traités dans la présente étude. Les productivités sont calculées à l'aide d'un modèle démographique à pas de temps annuel. Sa méthode consiste à comparer deux projections démographiques, avec et sans prophylaxie. Pour une projection donnée, les paramètres d'exploitation des femelles sont calculés lors de chaque exercice agricole pour assurer l'équilibre démographique stationnaire du cheptel. Les productivités sont calculées en cumulant les valeurs monétaires actualisées des animaux exploités sur l'ensemble des cinq exercices. Tillard ne présente pas de résultats comparatifs sur les productivités numériques. Seuls les rapports bénéfice/coût sont estimés, respectivement à 5,04 et 5,40 pour le fenbendazole et le morantel. Ces résultats sont difficiles à comparer avec ceux présentés dans cet article, du fait de la différence des modèles mis en œuvre et des méthodes utilisées pour le calcul des paramètres démographiques et du coût de la prophylaxie. Tillard estime les rapports bénéfice/coût d'après des valeurs monétaires actualisées et les coûts de traitement tiennent compte des coûts directs (prix d'achat des vermifuges) et indirects liés à la mise en place du projet. En revanche, dans la pré-

\section{Tableau II}

Prix de vente moyens (Fcfa) des ovins Djallonké de Kolda (Sénégal) par sexe, classe d'âge et période en 1996 (d'après Lesnoff et coll., 2000, Prev. vet. Med.)

\begin{tabular}{lrrrr} 
Classe d'âge & \multicolumn{2}{c}{ Femelles } & \multicolumn{2}{c}{ Mâles } \\
\cline { 2 - 6 } & HT $^{\mathbf{1}}$ & \multicolumn{1}{c}{$\mathbf{T}^{\mathbf{2}}$} & \multicolumn{1}{c}{ HT } & \multicolumn{1}{c}{ T } \\
\hline 0-3 mois & 5000 & 5000 & 5000 & 5000 \\
3-6 mois & 8500 & 8500 & 9500 & 10000 \\
6-12 mois & 10000 & 10000 & 12500 & 15500 \\
1-2 ans & 11500 & 11500 & 19000 & 27000 \\
$>$ 2 ans & 14000 & 14000 & - & -
\end{tabular}

1. Hors de la fête de la Tabaski

2. Lors de la fête de la Tabaski

\section{Tableau III}

Productivités numériques des ovins Djallonké de Kolda (Sénégal) estimées par groupe de traitement du plan de prophylaxie de 1987, sous I'hypothèse d'équilibre démographique stationnaire pour un effectif de 1000 animaux dans chaque groupe

\begin{tabular}{lccc}
$\begin{array}{l}\text { Groupes de } \\
\text { troupeaux }\end{array}$ & Femelles & Mâles & Total \\
\hline$W_{\mathrm{CC}}$ & 204,7 & 213,4 & 418,1 \\
$\mathrm{~W}_{\mathrm{CD}}$ & 259,4 & 238,4 & 497,6 \\
$\mathrm{~W}_{\mathrm{VC}}$ & 198,8 & 203,8 & 402,6 \\
$\mathrm{~W}_{\mathrm{VD}}$ & 240,7 & 265,4 & 506,1 \\
\end{tabular}

$C C$ : non vacciné et non vermifugé ; $C D$ : non vacciné et vermifugé ; $\mathrm{VC}$ : vacciné et non vermifugé ; VD : vacciné et vermifugé

\section{Tableau IV}

Rapports des productivités numériques totales (femelles et mâles) des ovins Djallonké de Kolda (Sénégal) estimées par groupe de traitement du plan de prophylaxie de 1985-1987, sous I'hypothèse

d'équilibre démographique stationnaire, pour un effectif de 1000 animaux par groupe

\begin{tabular}{lc} 
Effet & Rapport des productivités * \\
\hline Vaccination & \\
$\mathrm{W}_{\mathrm{VC}} / \mathrm{W}_{\mathrm{CC}}$ & 0,911 \\
$\mathrm{~W}_{\mathrm{VD}} / \mathrm{W}_{\mathrm{CD}}$ & 0,952 \\
$\left(\mathrm{~W}_{\mathrm{VC}}+\mathrm{W}_{\mathrm{VD}}\right) /\left(\mathrm{W}_{\mathrm{CC}}+\mathrm{W}_{\mathrm{CD}}\right)$ & 0,934 \\
Vermifugation & \\
$\mathrm{W}_{\mathrm{CD}} / \mathrm{W}_{\mathrm{CC}}$ & 1,245 \\
$\mathrm{~W}_{\mathrm{VD}} / \mathrm{W}_{\mathrm{VC}}$ & 1,301 \\
$\left(\mathrm{~W}_{\mathrm{CD}}+\mathrm{W}_{\mathrm{VD}}\right) /\left(\mathrm{W}_{\mathrm{CC}}+\mathrm{W}_{\mathrm{VC}}\right)$ & 1,272
\end{tabular}

* Un rapport supérieur à 1 indique un effet positif ; un rapport inférieur à 1 indique un effet négatif

$\mathrm{CC}$ : non vacciné et non vermifugé $; \mathrm{CD}$ : non vacciné et vermifugé ; $\mathrm{VC}$ : vacciné et non vermifugé ; VD : vacciné et vermifugé 
sente étude, le rapport bénéfice/coût a été calculé sur un seul exercice agricole de projection et le coût n'a pris en compte que le prix d'achat du vermifuge. Ce choix a semblé refléter les dépenses des éleveurs dans les conditions rencontrées à Kolda lors de l'étude (29).

Dans la présente étude, la vaccination contre la pasteurellose n'a pas montré d'effet positif sur la productivité numérique (aucun effet n'a été observé par Tillard ; 43). Elle a même présenté un effet négatif chez les femelles pour le plan 1987 et chez l'ensemble des animaux pour le plan 1985-1987 (sous réserve des tests de significativité non effectués). Cet effet paradoxal pourrait provenir de facteurs biologiques ou expérimentaux, mais il n'a pas été expliqué. De nouvelles expérimentations seront nécessaires pour évaluer plus précisément l'effet du vaccin contre la pasteurellose respiratoire sur la productivité des ovins.

\section{Méthode de calcul de la productivité}

\section{Comparaison avec la méthode démographique conventionnelle}

Notre méthode a un principe différent de celui des modèles de production à l'équilibre conventionnels (46). Les deux méthodes calculent la productivité d'un cheptel d'effectif total $\mathrm{n}$ en début de projection, pour une hypothèse de croissance démographique et une hypothèse fixant la pratique de gestion des effectifs du cheptel. Mais elles diffèrent dans la représentation de cette pratique de gestion : la structure d'exploitation dans notre méthode et la structure d'âge et de sexe dans la méthode conventionnelle. Dans les modèles conventionnels, l'effectif des animaux exploitables est calculé par rapport à une structure d'âge et de sexe (proportions des différentes classes d'âge de femelles et de mâles) v fixée comme objectif pour le cheptel. Dans le cas d'un pas de temps annuel et d'un cheptel de femelles à trois classes d'âge (voir plus haut), dans l'hypothèse d'équilibre stationnaire, les probabilités d'exploitation $\left\{\mathrm{P}_{0}(\exp )\right.$, $\left.\mathrm{P}_{1}(\exp ), \mathrm{P}_{2}(\exp ), \mathrm{P}_{3}(\exp )\right\}$ sont calculées en résolvant le système linéaire $x(t)=A x(t)$. Les fécondités, les probabilités de mort naturelle et le vecteur d'état $\mathrm{x}(\mathrm{t})=\mathrm{n}$ v sont fixés (les seules inconnues à déterminer sont les probabilités d'exploitation). Lorsque plusieurs cheptels sont comparés, le même vecteur d'état $\mathrm{x}(\mathrm{t})$ est choisi comme référence pour tous les cheptels.

La structure d'exploitation et la structure d'âge et de sexe représentent chacune une contrainte dans le modèle démographique et ne peuvent être fixées simultanément. Supposons que deux cheptels soient comparés. Dans notre méthode, la productivité est calculée après avoir fixé une structure d'exploitation identique pour les deux cheptels. En situation d'équilibre, les deux cheptels vont présenter une structure d'âge et de sexe différente, résultat du calcul démographique (l'analyse de plusieurs jeux de données, non présentée ici, semble cependant indiquer que ces structures varient peu en pratique). En revanche, dans la méthode conventionnelle, la productivité est calculée après avoir fixé le même vecteur $\mathrm{x}(\mathrm{t})$ pour les deux cheptels. En situation d'équilibre, les deux cheptels vont donc présenter la même structure d'âge et de sexe, mais leur structure d'exploitation sera différente, puisqu'elle est le résultat du calcul démographique.

Les deux méthodes sont complémentaires et leur emploi dépend des objectifs poursuivis : contrôle de la structure d'exploitation ou de la structure d'âge et de sexe. Elles sont très proches dans le cas d'un modèle à pas de temps annuel. Notre approche présente cependant l'avantage d'être facilement applicable au modèle démographique par quinzaine. Une fois que la structure d'exploitation de référence est fixée, le problème est ramené au simple calcul de l'intensité d'exploitation $\varphi_{\text {objectif }}$ par une méthode itérative.

\section{Hypothèses posées dans le calcul de la productivité}

Dans la présente étude, les productivités W ont été calculées en situation d'équilibre démographique stationnaire et conditionnellement à la structure d'exploitation nette estimée d'après les données. D'autres choix sont possibles et influent sur les résultats d'analyse comparative. L'analyse de sensibilité présentée plus haut a montré, par exemple, que les rapports de productivité pouvaient varier avec le taux de croissance démographique fixé comme hypothèse. Des études effectuées sur d'autres jeux de données (non présentées dans cet article) ont montré que la structure d'exploitation nette pouvait également modifier les résultats. Les hypothèses sous-jacentes aux calculs de productivité (hypothèses de croissance démographique et de pratique de gestion des effectifs des cheptels) doivent donc être décrites précisément dans les analyses comparatives. Dans le cas contraire, la comparaison des diagnostics entre études peut devenir difficile à interpréter. Par rapport à d'autres méthodes plus empiriques, notre méthode, et plus généralement la méthode des modèles de production à l'équilibre, permet de présenter clairement ces hypothèses et d'étudier l'effet de leur variation sur les résultats (analyse de sensibilité).

La productivité d'un cheptel peut être définie comme le rapport entre les " quantités produites » (numérateur) et les " moyens de production mis en œuvre » (dénominateur) qui doit refléter respectivement les objectifs de l'éleveur et ses contraintes les plus limitantes $(8,46)$. Dans la présente étude, ces moyens de production ont été représentés par l'effectif total $\mathrm{n}$ des cheptels en début de projection. Notre méthode, cependant, permet de calculer d'autres indicateurs classiques de productivité, comme la productivité numérique au sevrage (30). Pour cette productivité au sevrage, par exemple, les moyens de production sont représentés par l'effectif des femelles reproductrices présentes dans les différents cheptels, en début d'année ou en moyenne sur l'année. Cet effectif est calculé directement d'après les sorties du modèle. Les résultats concernant la productivité au sevrage des cheptels d'ovins dans la région de Kolda n'ont pas été présentés dans cette étude car ils n'ont pas modifié les diagnostics obtenus sur l'efficacité des traitements.

Par ailleurs, notre méthode permet de calculer des indicateurs plus complexes prenant en compte des mesures énergétiques $(4,8,46)$. $\mathrm{Si}$ les données sont disponibles, le calcul de tels indicateurs s'effectue aisément (le modèle permet de connaître à chaque quinzaine de l'année l'effectif des femelles et des mâles pour chaque classe d'âge) et sans modifier l'algorithme défini dans la méthode (seul le calcul du dénominateur change).

Enfin, dans notre méthode, le scalaire $\varphi$ contrôlant l'intensité d'exploitation ne concerne que les femelles dont le taux de croissance détermine celui des mâles. Lorsque $\varphi$ augmente, les paramètres d'exploitation nette des mâles restent constants. Ils doivent donc représenter correctement les niveaux d'exploitation nette observés dans les cheptels. Pour les plans de prophylaxie de 1987 et de 1985-1987, la structure mâle de référence a été définie par la moyenne des probabilités d'exploitation nette calculée sur l'ensemble des cheptels et des exercices agricoles étudiés. Cette procédure a donné de bons résultats pour la prédiction des structures d'âge et de sexe des cheptels (28). Elle paraît bien adaptée aux populations d'ovins en milieu traditionnel pour lesquelles l'hypothèse de femelle-dominance est cohérente (27). Des modèles démographiques permettant de contrôler l'exploitation des deux sexes ont cependant été proposés pour certaines populations sauvages de grands mammifères (31). Ils mettent en jeu des paramètres de reproduction densité-dépendants. Il reste à évaluer l'intérêt de tels modèles, plus complexes $(12,20)$, dans le cadre des cheptels domestiques. 


\section{Discrétisation du temps}

Le pas de temps annuel pose des problèmes importants pour le calcul de la productivité de cheptels tels que les petits ruminants.

Tout d'abord, il engendre une décomposition du cheptel en classes d'âges annuelles ou pluriannuelles. Pour les petits ruminants, ces classes sont hétérogènes (par exemple, la classe d'âge 0-1 an regroupe des jeunes non sevrés et des animaux presque adultes). L'interprétation des paramètres démographiques associés à ces classes d'âge est difficile.

Ensuite, il ne permet pas d'intégrer la variabilité saisonnière des paramètres démographiques ou d'autres paramètres pouvant intervente des animaux. Ce défaut peut biaiser l'estimation de la productivité des cheptels.

Enfin, il accroît les biais des estimations de la productivité dus au caractère mutuellement exclusif de certains événements démographiques, par exemple la mortalité naturelle et l'exploitation. La théorie des risques compétitifs montre que la probabilité de mort naturelle des animaux d'un cheptel dans une période donnée est une fonction décroissante de son niveau d'exploitation dans cette même période $(1,10)$ : pour des valeurs pas trop élevées de $\mathrm{P}_{\mathrm{i}}$ (mor) et $\mathrm{P}_{\mathrm{i}}(\exp )$, cette relation est $\mathrm{P}_{\mathrm{i}}($ mor $) \approx \mathrm{P}^{*}{ }_{\mathrm{i}}($ mor $)-\mathrm{P}^{*}{ }_{\mathrm{i}}($ mor $) \mathrm{P}_{\mathrm{i}}(\exp )$, où $\mathrm{P}^{*}$ (mor) représente la probabilité de mort naturelle observée dans le cheptel lorsqu'il n'y a pas d'exploitation (1). En d'autres termes, pour un même risque journalier de mort naturelle, plus le cheptel est exploité, plus la mortalité apparente $\mathrm{P}_{\mathrm{i}}$ (mor) est faible : l'exploitation est un facteur de confusion pour la mortalité naturelle. Ces interférences statistiques entre les paramètres $(25,28,37)$ touchent également la fécondité et prennent d'autant plus d'importance que la compétition entre événements démographiques est forte et que la durée de la période est grande. Ce problème, déjà souligné par Landais (25), ne peut être éludé pour les cheptels soumis à une forte exploitation (petits ruminants ou autres cheptels) car les biais engendrés peuvent donner lieu à des erreurs d'interprétation dans les analyses comparatives. Cependant, sa prise en compte se limite souvent à corriger l'estimation de la mortalité naturelle par la méthode des tables de vie $(3,34,43)$ et les interférences ne sont pas prises en compte pour la fécondité. Outre les biais d'estimation, les interférences statistiques posent un problème dans la définition des modèles démographiques à pas de temps annuel : par exemple la décomposition $\mathrm{s}_{\mathrm{i}}=1-\mathrm{P}_{\mathrm{i}}(\mathrm{mor})-\mathrm{P}_{\mathrm{i}}(\exp )$ est inadaptée puisqu'il est incorrect de faire varier l'exploitation $\mathrm{P}_{\mathrm{i}}(\exp )$ sans faire varier la mortalité apparente $\mathrm{P}_{\mathrm{i}}(\mathrm{mor})$. Pour contourner ce problème, les modèles supposent souvent que l'exploitation est instantanée et survient en fin d'année (14) : la probabilité de survie est définie par $\mathrm{s}_{\mathrm{i}}$ $=\left(1-h_{i}\right)\left[1-P^{*}{ }_{i}(m o r)\right]$, où $h_{i}$ représente la fraction exploitée en fin d'année. Cette hypothèse élimine la possibilité d'interférences (si les probabilités $\mathrm{P}_{\mathrm{i}}^{*}$ (mor) sont convenablement estimées), mais elle ne respecte pas la distribution continue dans le temps de l'exploitation des animaux observée dans les cheptels domestiques tropicaux.

Le modèle démographique proposé dans cette étude a permis de répondre aux trois limitations précédentes. En particulier pour les cheptels d'ovins, le regroupement des données par quinzaine a protégé des interférences statistiques (28) : les variations de fécondité ou de mort naturelle observées au sein des quinzaines n'ont pu être dues à des variations du niveau d'exploitation des cheptels, excepté dans certains cas pour les mâles d'âge supérieur à un an (pour éliminer ce problème dans le calcul de la productivité, nous avons supposé dans la structure d'exploitation de référence que tous les mâles étaient réformés à l'âge d'un an, situation proche des pratiques de gestion observées sur le terrain).
Le choix du pas de temps influe sur le protocole d'enquête de terrain et sur le rythme de passage des enquêteurs dans les élevages. Dans le cas de cette étude, les événements démographiques (naissances, morts, entrées ou sorties du troupeau) ont été identifiés au moins à la quinzaine près. Les suivis d'élevages avec marquage individuel des animaux (18) ont l'avantage de fournir ce type de données avec fiabilité. En revanche, les données produites sont souvent de natures diverses, touchent plusieurs niveaux d'observation (animal ou troupeau) et leur gestion, complexe, nécessite des outils adaptés (21).

L'un des problèmes pratiques rencontré par le zootechnicien lorsqu'il met en place un suivi d'élevages est de savoir quel rythme de passage imposer aux enquêteurs, l'enjeu étant d'alléger le plus possible le protocole de terrain. Ce type de question peut en réalité avoir des réponses très différentes selon l'espèce et le phénomène que l'on souhaite observer. L'examen de certains signes pathologiques sur les animaux, comme le jetage, demande par exemple un passage presque journalier dans les élevages. Pour la démographie, l'augmentation du pas de temps engendre deux sources de biais : les interférences entre les événements démographiques et la capacité de l'éleveur à se remémorer (et transmettre à l'enquêteur) l'ensemble des événements survenus entre deux passages. Dans la présente étude, nous n'avons pas cherché à quantifier systématiquement l'effet de la durée du pas de temps, en considérant une gamme de variation donnée, sur la force des interférences. Les niveaux d'exploitation en général assez élevés des petits ruminants limitent cependant, selon nous, les possibilités de choisir un pas de temps nettement supérieur à la quinzaine, sans introduire rapidement des biais importants dans l'estimation des paramètres (il est d'ailleurs à noter que la quinzaine a été retenue comme pas de temps dans différentes applications sur la démographie des ovins ; $6,13)$. En outre, compte tenu du rythme élevé des événements démographiques observés dans les troupeaux de petits ruminants, il est vraisemblable qu'un pas de temps trop grand augmenterait significativement les erreurs de mesures lors des enquêtes de terrain (oublis d'événements) et les biais qui en découlent. Pour des espèces comme les bovins, présentant un cycle de reproduction plus long ainsi que des niveaux d'exploitation et des rythmes d'événements démographiques plus faibles, le pas de temps pourrait en revanche sûrement être augmenté.

\section{CONCLUSION}

Par rapport aux modèles conventionnels de production à l'équilibre pour l'analyse comparative de la productivité des cheptels, la méthode proposée a apporté trois améliorations importantes :

- en présence d'événements démographiques mutuellement exclusifs (risques compétitifs), la discrétisation du temps en phases courtes a permis d'éliminer les interférences statistiques entre les paramètres démographiques. Cela a limité les biais dans l'estimation des paramètres démographiques et de la productivité ;

- le modèle a permis une représentation fidèle de la dynamique intra-annuelle et interannuelle des cheptels. De manière imagée, le modèle par quinzaine a permis de « superposer » les distributions bidimensionnelles (classes d'âge x quinzaines dans l'année) des paramètres de mort naturelle, de fécondité, d'exploitation et d'importation, ainsi que d'autres paramètres comme le poids ou le prix de vente des animaux. Le modèle a fait la synthèse de l'ensemble des interactions existant entre ces distributions et a intégré l'effet des positions relatives de phénomènes naturels (par exemple, pics de mise bas, pics de mortalité en saison des pluies) ou commerciaux (par exemple, l'augmentation du prix de vente des animaux lors de la fête de la Tabaski). Le modèle a pris en compte les fluctuations saisonnières des structures d'âge et de l'effectif total des populations. Ces propriétés sont importantes 
pour les petits ruminants des systèmes extensifs tropicaux, entièrement dépendants des grands changements dans l'environnement engendrés notamment par la succession des saisons ;

- la méthode d'inférence proposée, utilisant une procédure de bootstrap non paramétrique, a été simple à mettre en œuvre et a permis de calculer des intervalles de confiance et de réaliser des tests statistiques pour les productivités estimées.

La méthode peut être appliquée à d'autres espèces domestiques ou sauvages que les petits ruminants, dans des études zootechniques (pour évaluer par exemple l'effet de différentes pratiques d'élevage), épidémiologiques ou écologiques. Elle peut être utilisée au sein de programmes de recherche, mais également par des responsables de projets de développement souhaitant évaluer l'efficacité des actions d'amélioration proposées. La mise en œuvre complète du modèle démographique par quinzaine nécessite de disposer de données de suivis d'élevages, planifiés en fonction des objectifs de ces programmes ou projets. La répartition cohérente des élevages suivis dans les différentes modalités à tester (pratiques d'élevage, régimes alimentaires, traitements sanitaires ou autres critères) est une condition importante pour l'application de la méthode.

La méthode est très souple. La facilité de construction d'une large gamme de structures d'exploitation nette permet de s'adapter aux stratégies d'exploitation rencontrées en élevage tropical et d'étudier la sensibilité des analyses comparatives à ces différentes stratégies. Les techniques d'analyses de sensibilité (non présentées ici) permettent par ailleurs de quantifier la contribution respective des différents paramètres démographiques, regroupés par exemple par groupe d'âge, par saison ou par type d'événement (fécondité, mortalité) dans les écarts de productivités observés entre les cheptels $(12,28)$.

Enfin, différentes extensions du modèle démographique sont possibles, par exemple en augmentant la diversité des états individuels représentés dans le modèle (parité des femelles, taille des portées, statut nutritionnel ou statut épidémiologique). Le modèle peut également être utilisé dans d'autres contextes tels que les études d'optimisation pour identifier les stratégies d'exploitation les plus pertinentes par rapport à un objectif fixé. Ces développements seront présentés dans des travaux ultérieurs.

\section{Remerciements}

Nous remercions l'Institut sénégalais de recherches agricoles, Laboratoire national de recherche vétérinaire (Isra-Lnerv), la Direction de l'élevage au Sénégal et les éleveurs de la région de Kolda pour leur collaboration. Nous remercions Olivier et Brigitte Faugère pour la qualité des données qu'ils nous ont fournies. Enfin, nous tenons à remercier les trois lecteurs anonymes qui ont permis d'améliorer notre article par leurs remarques pertinentes.

\section{BIBLIOGRAPHIE}

1. ANDERSON D.R., BURNHAM K.P., 1976. Population of the Mallard. VI. The effect of exploitation on survival. Washington DC, USA, Department of the Interior, Fish and Wildlife Service, 66 p. (Resource Publication 128)

2. ANKERS P., ITTY P., ZINSSTAG J., TRAWALLY S., PFISTER K., 1998. Biannual anthelmintic treatments in village Djallonke sheep in the Gambia: effects on productivity and profitability. Prev. vet. Med., 34: 215-225.

3. AWA D.N., NJOYA A., NGOTAMA A.C., 2000. Economics of prophylaxis against peste de petits ruminants and gastrointestinal helminthosis in small ruminants in North Cameroun. Trop. Anim. Health Prod., 32: 391-403.

4. BAPTIST R., 1992. Derivation of steady-state herd productivity. Agric. Syst., 39: 253-272.

5. BLACKBURN H.D., BRYANT F.C., CARTWRIGHT T.C., FIERRO L.C., 1989. Corriedale sheep production when supplemented with ryegrass pasture. Agric. Syst., 30: 101-115.

6. BLACKBURN H.D., CARTWRIGHT T.C., 1987. Description and validation of the Texas AM sheep simulation model. J. Anim. Sci., 67: 373-386.
7. BOSMAN H.G., AYANTUNDE A.A., STEENSTRA F.A., UDO H.M., 1997. A simulation model to assess productivity of goat production in the tropics. Agric. Syst., 54: 539-576.

8. BOSMAN H.G., MOLL H.A.J., UDO H.M.J., 1997. Measuring and interpreting the benefits of goat keeping in tropical farm systems. Agric. Syst., 53: 349-372.

9. BRAULT S., CASWELL H., 1993. Pod-specific demography of Killer Whales (Orcinus orca). Ecology, 74: 1444-1454.

10. BURNHAM K.P., ANDERSON D.R., 1984. Tests of compensatory vs additive hypotheses of mortality in mallards. Ecology, 65: 105-112.

11. BURNHAM K.P., ANDERSON D.R., 1998. Model selection and inference. A practical information-theoretic approach. New York, NY, USA Springer.

12. CASWELL H., 1989. Matrix population models. Construction, analysis and interpretation. Sunderland, MA, USA, Sinauer Associates, $321 \mathrm{p}$.

13. DEDIEU B., COURNUT S., 2000. Le fonctionnement d'un troupeau ovin viande : conception d'un modèle de simulation par événements discrets. In : Séminaire Inra-Cirad Modélisation du fonctionnement des troupeaux, Theix, France, 31 août-1er septembre 2001, p. 45-54.

14. DOUBLEDAY W.G., 1975. Harvesting in matrix population. Biometrics, 31: 189-200.

15. EFRON B., TIBSHIRANI R.J., 1993. An introduction to the bootstrap. London, UK, Chapman \& Hall, $436 \mathrm{p}$.

16. FAUGERE O., DOCKES A.C., PERROT C., FAUGERE B., 1990. L'élevage traditionnel des petits ruminants au Sénégal. I. Pratiques de conduite et d'exploitation des animaux chez les éleveurs de la région de Kolda. Revue Elev. Méd. vét. Pays trop., $43:$ 249-259.

17. FAUGERE O., DOCKES A.C., PERROT C., FAUGERE B., 1990. L'élevage traditionnel des petits ruminants au Sénégal. II. Pratiques de conduite et d'exploitation des animaux chez les éleveurs de la région de Louga. Revue Elev. Méd. vét. Pays trop., $43:$ 261-273

18. FAUGERE O., FAUGERE B., 1986. Suivi de troupeaux et contrôle des performances individuelles des petits ruminants en milieu traditionnel africain. Aspects méthodologiques. Revue Elev. Méd. vét. Pays trop., 39 : 29-40.

19. FAUGERE O., TILLARD E., FAUGERE B., 1992. Prophylaxie chez les petits ruminants au Sénégal : régionalisation d'une politique nationale de protection sanitaire. In : Ilrad, Ed., First biennial conference of the African Small Ruminant Research Network, Nairobi, Kenya, ILCA, p. 307-314.

20. GETZ W.M., HAIGHT R.G., 1989. Population harvesting: demographic models of fish, forest, and animal resources. Princeton, NJ, USA, Princeton University Press, $381 \mathrm{p}$.

21. JUANES X., LANCELOT R., 1999. Laser : Logiciel d'aide au suivi d'élevage des ruminants. Montpellier, France, Cirad-emvt.

22. KALISZ S., MCPEEK M.A., 1992. Demography of an age-structured annual: resampled projection matrices, elasticity analyses, and seed bank effects. Ecology, 73: 1082-1093.

23. KNIPSCHEER H.C., KUSNADI O., DE BOER A.J., 1984. Some efficiency measures for analysis of the productive potential of Indonesian goats. Agric. Syst., 15: 125-135

24. LANCELOT R., FAYE B., JUANES X., NDIAYE M., PEROCHON L., TILLARD E., 1998. La base de données Baobab : un outil pour modéliser la production et la santé des petits ruminants dans les systèmes d'élevage traditionnels au Sénégal. Revue Elev. Méd. vét. Pays trop., 51 : 135-146.

25. LANDAIS E., 1986. Contributions à l'étude des interférences entre phénomènes démographiques et propositions pour l'estimation sans biais des paramètres démographiques usuels. In : Landais E., Fave J. ed., Méthodes pour la recherche sur les systèmes d'élevage en Afrique intertropicale. Maisons-Alfort, France, Cirad-lemvt, p. 497-517. (Etudes et synthèses de I'lemvt, $\left.n^{\circ} 20\right)$

26. LESLIE P.H., 1945. On the use of matrices in certain population mathematics. Biometrika, 33: 183-212.

27. LESNOFF M., 1999. Dynamics of a sheep population in a Sahelian area (Ndiagne district in Senegal): a periodic matrix model. Agric. Syst., 61 207-221.

28. LESNOFF M., 2000. Etude de la dynamique et de la productivité des populations domestiques tropicales par les modèles matriciels en temps discret : les populations d'ovins au Sénégal. Thèse Doct., Université de Montpellier II, Montpellier, France. 
29. LESNOFF M., LANCELOT R., TILLARD E., DOHOO I.R., 2000. A steadystate approach of benefit-cost analysis with a periodic Leslie-matrix model. Presentation and application to the evaluation of a sheep-diseases preventive scheme in Kolda, Senegal. Prev. vet. Med., 46: 113-128.

30. LHOSTE P., DOLLE V., ROUSSEAU J., SOLTNER D., 1993. Manuel de zootechnie des régions chaudes - Les systèmes d'élevage. Paris, France, ministère de la Coopération, 288 p. (Coll. Précis d'élevage)

31. LINDSTROM J., 1998. Harvesting and sex differences in demography. Wild. Biol., 4: 213-221.

32. MATHSOFT, 1999. Splus 2000 professionnal, release 1. Seattle, WA USA, MathSoft.

33. MEYER J.S., INGERSOLL C.G., MCDONALD L.L., BOYCE M.S., 1986 Estimating uncertainty in population growth rates: jacknife vs. bootstrap techniques. Ecology, 67: 1156-1166.

34. MOULIN C.-H., 1993. Performances animales et pratiques d'élevage en Afrique sahélienne. La diversité du fonctionnement des troupeaux de petits ruminants dans la Communauté rurale de Ndiagne (Sénégal). Thèse Doct., Ina-Pg, Paris, France, 259 p.

35. NDAMUKONG K.J.N., SEWELL M.M.H., ASANJI M.F., 1989 Management and productivity of small ruminants in the North West Province of Cameroon. Trop. Anim. Health Prod., 21: 109-119.

36. PEACOCK C.P., 1987. Measures for assessing the productivity of sheep and goats. Agric. Syst., 23: 197-210.

37. PRESSAT R., 1983. L'analyse démographique. Concepts, méthodes, résultat. Paris, France, Presses universitaires de France, 295 p.

38. PUTT S.N.H., SHAW A.P.M., WOODS A.J., TYLER L., JAMES A.D. 1987. Veterinary epidemiology and economics in Africa. A manual for use in the design and appraisal of livestock health policy. Addis Ababa, Ethiopia, ILCA, p. 111-122. (Manual 3)

39. SANDERS J.O., CARTWRIGHT T.C., 1979. A general cattle production systems model. I. Structure of the model. Agric. Syst., 4: 217-227.

40. SKELLAM J.G., 1967. Seasonal periodicity in theoretical population ecology. In: Proc. 5th Berkeley Symp., Berkeley, USA, Vol. IV, p. 179-205.

41. TACHER G., 1975. Note sur la démographie bovine au Sahel. I. Représentation et projection théorique du troupeau par un modèle mathématique. Revue Elev. Méd. vét. Pays trop., 28 : 547-569.

42. THE MATH WORKS, 1999. Matlab, version 5.3. Natick, MA, USA, The Math Works.

43. TILLARD E., 1991. Evaluation technico-économique des effets de prophylaxies en milieu villageois chez les petits ruminants de la région de Kolda (Sénégal). Thèse Doct. vét., Ecole nationale vétérinaire de MaisonsAlfort, Université de Créteil, France, 172 p.

44. TILLARD E., MOULIN C.-H., FAUGERE O., FAUGERE B., 1997. Le suivi individuel des petits ruminants au Sénégal: un mode d'étude des troupeaux en milieu villageois. Inra Prod. Anim., 10 : 67-78.

45. TULJAPURKAR S., CASWELL H., 1997. Structured-population models in marine, terrestrial, and freshwater systems. New York, NY, USA, Chapman and Hall, 643 p.

46. UPTON M., 1989. Livestock productivity assessment and herd growth models. Agric. Syst., 29: 149-164.

47. WILSON R.T., 1983. Studies on the livestock of Southern Darfur, Sudan. VIII. A comparison of productivity indices for goats and sheep. Trop. Anim. Health Prod., 15: 63-68.

Reçu le 03.01.2001, accepté le 10.07.2001

\section{Summary}

Lesnoff M., Lancelot R., Tillard E., Faye B. Comparative Analysis of the Productivity of Small Ruminant Flocks under Tropical Extensive Management: A New Leslie-Matrix Model Approach

A new method for the comparative analysis of productivity of tropical domestic flocks is presented here. This method used discrete-time demographic matrix models and the steady-state approach. Conventional steady-state demographic models use an annual step-time, which is poorly adapted to species with relatively short breeding cycles and with parturition occurring throughout the year. In the new model, we divided the year into two-week periods. The three major operational advantages were as follows: first, the short step-time that could decrease the bias generated by competing risks in the estimated demographic parameters (fecundity, mortality, animal outflow or inflow); second, the periodic model that could represent both the intraannual and interannual variations in demographic parameters and in other parameters like the live weight or the sale price of animals; finally, a proposed inference method, which used non parametric bootstrap, that helped calculate confidence intervals and build tests to compare the productivity of various flocks. The method was tested on field data obtained from sheep flocks in Senegal. It can also be applied to other domestic species and to wild species in various zootechnical or ecological environments.

Key words: Sheep - Population dynamics - Productivity - Mathematical model - Senegal.

\section{Resumen}

Lesnoff M., Lancelot R., Tillard E., Faye B. Análisis comparativo de la productividad de los hatos de pequeños rumiantes en crianza tropical extensiva: un nuevo enfoque mediante modelos de matrices en tiempos discretos

El objetivo del presente artículo es el de presentar un método de análisis comparativo de la productividad de los hatos domésticos tropicales. El método propuesto utiliza modelos demográficos de matrices y es parte de los modelos de producción en equilibrio (steady state models). Los métodos clásicos utilizan modelos a tiempo anual, poco adaptados para las especies con ciclos reproductivos relativamente cortos y con partos distribuidos a lo largo del año. Nuestro método utiliza un modelo que desglosa el año en quincenas, permitiendo representar conjuntamente las variaciones intra e inter anuales de los parámetros demográficos (fecundidad, mortalidad natural, explotación o importación de animales) y ponderados (peso o precio de venta de los animales). Se presentan tres aportes operacionales con respecto a los enfoques clásicos. La descomposición del tiempo en quincenas permite limitar los fenómenos de interferencia estadística entre los parámetros demográficos y las desviaciones en las productividades estimadas y de respetar la estructura estacional de los procesos encontrados en la cría. Finalmente, el método de inferencia propuesto (utilizando el bootstrap no paramétrico) permite calcular los intervalos de confianza y realizar las pruebas estadísticas para las productividades estimadas. El método se realiza con datos de campo colectados en hatos ovinos de Senegal. El método propuesto puede aplicarse directamente a otras especies domésticas o silvestres, en contextos zootécnicos o ecológicos diversos.

Palabras clave: Ovino - Dinamica de poblaciones - Productividad - Modelo matematico - Senegal. 